\title{
Lorentz-covariant quantum mechanics and preferred frame
}

\author{
P. Caban' and J. Rembieliński² \\ Department of Theoretical Physics, University of Łódź, \\ Pomorska 149/153, 90-236 Łódź, Poland
}

$2018-7-8$

\begin{abstract}
In this paper the relativistic quantum mechanics is considered in the framework of the nonstandard synchronization scheme for clocks. Such a synchronization preserves Poincaré covariance but (at least formally) distinguishes an inertial frame. This enables to avoid the problem of a noncausal transmision of information related to breaking of the Bell's inequalities in QM. Our analysis has been focused mainly on the problem of existence of a proper position operator for massive particles. We have proved that in our framework such an operator exists for particles with arbitrary spin. It fulfills all the requirements: it is Hermitean and covariant, it has commuting components and moreover its eigenvectors (localised states) are also covariant. We have found the explicit form of the position operator and have demonstrated that in the preferred frame our operator coincides with the Newton-Wigner one. We have also defined a covariant spin operator and have constructed an invariant spin square operator. Moreover, full algebra of observables consisting of position operators, fourmomentum operators and spin operators is manifestly Poincaré covariant in this framework. Our results support expectations of other authors (Bell [月], Eberhard [8]) that a consistent formulation of quantum mechanics demands existence of a preferred frame.
\end{abstract}

\footnotetext{
${ }^{1}$ E-mail address: caban@mvii.uni.lodz.pl

${ }^{2}$ E-mail address: jaremb@mvii.uni.lodz.pl and jaremb@krysia.uni.lodz.pl
} 


\section{Introduction}

In this paper we propose a formulation of the Poincaré covariant quantum mechanics for a free particle. Our investigations were motivated by two old and still open problems: violation of locality in quantum mechanics (breaking of Bell's inequalities) and nonexistence of a covariant position operator as well as covariant localized states. It was recognised long time ago, that some correlation experiments (like Aspect et al [1, 2, 3] ones) imply, that, as was stressed by H. P. Stapp 21] "... what happens macroscopically in one space-time region must in some cases depend on variables that are controlled by experimenters in far-away, space-like-separated regions". This fact can be in a conflict with special relativity; even more frustrating is a conflict with a causal transmission of information. It may be interesting to recall in this place the statement by $\mathrm{J}$. S. Bell [6]: "... For me then this is the real problem with quantum theory: the apparently essential conflict between any sharp formulation and fundamental relativity". According to Bell [5] (see also Eberhard [8]) consistent formulation of relativistic quantum mechanics may be necessarily with a preferred frame at the fundamental level. Following these suggestions we try to construct here a quantum mechanics which has built-in the preferred frame and which is at the same time Poincaré covariant. It is important to realise at this point, that special relativity is in fact based on two main assumptions: Poincaré covariance and relativity principle. So the key point is to reformulate this theory in a way which preserves the Poincaré covariance but abandons the relativity principle and consequently allows us to introduce a preferred frame. Such a formulation of relativity theory has been given by one of the authors (J.R.) in [18, 19]. It was shown there, that, using a nonstandard synchronization procedure for clocks (named in 19] as the Chang-Tangherlini synchronization), it is possible to obtain such a form of the transformations of coordinates beetwen inertial observers, that they realize Lorentz transformations, the time coordinate is only rescaled by a positive factor and the space coordinates do not mix to it. A price for this is existence of a preferred frame in the theory and dependence of Lorentz group transformations on the additional parameter - the fourvelocity of the preferred frame. Usually it is claimed that existence of a preferred frame violates the Poincaré covariance. This is really the case when we restrict ourselves to the Minkowski space-time. But in our approach the additional set of parameters (the mentioned above fourvelocity) allows us to solve this difficulty. That is this theory preserves Poincaré covariance but not necessarily the relativity principle. However, in our opinion, this is not any serious problem: in the real (expanding) Universe such a frame really does exist - it is the so called comoving frame related to the matter and the cosmic background radiation frame. Furthermore, in our framework, average light velocity over closed paths is still constant and equals to $c$, so Michelson-Morley like experiments do not distinguish such a possibility from the standard one [12, 19]. Moreover, in the context of the Poincaré covariant quantum mechanics presented herein, the 
above mentioned conflict between causality and quantum theory disappears. We also hope, that this formulation will be very convenient in the case of Poincaré covariant de Broglie-Bohm approach to quantum mechanics ${ }^{3}$. Next problem which is solved in this context is the localisation problem. Various aspects of localizability of particles have been studied from the early days of quantum mechanics, but, in the relativistic case (in contradiction to the non-relativistic one) the fully satisfactory position operator has not been found up to now. Let us explain at this point what we mean by the satisfactory position operator. Such an operator should be Hermitean, have commuting components (for massive particles), fulfill the canonical commutation relations with the momentum operators, be covariant and have covariant eigenstates (localized states). Operator constructed in the framework of the presented here theory fulfills all of the stated above conditions. To make this paper self-contained we devote the second section to the description of the Chang-Tangherlini synchronization scheme and the corresponding Lorentz group realization. More details on this subject one can find in [19]. In the section 3, following [19, 20], we describe briefly the covariant canonical formalism for a relativistic free particle on the classical level. The section 4 contains description of the quantum theory in the Chang-Tangherlini synchronization and the definition of the position operator. In the section 5 we construct and classify unitary orbits of the Poincaré group using the introduced position operator. We define also covariant spin operator and construct the invariant operator of the spin square. In the section 6 we firstly give a very brief review of the properties of the Newton-Wigner operator (in the authors' opinion the best position operator which has been constructed up to now; more information about the history of the localization problem and the full bibliography can be found in 14,15 ). Then we find the explicit form of our position operator in the functional realization and show that in the preferred frame our operator coincides with the Newton-Wigner one. This section contains also a discussion of the position operator under the special choice of the integral measure. In this case the form of the position operator is the same as in the nonrelativistic quantum mechanics.

\section{The Chang-Tangherlini (CT) synchronization}

In this chapter we briefly describe main results connected with the CT synchronization scheme which we shall use in the following. Derivation of these results one can find in 19]. The idea applied there is based on well known facts that the definition of the time coordinate depends on the choice of the synchronization scheme for clocks and that this choice is a convention [12, 13, 17, 22, 23. Using this freedom of choice one can try to find a synchronization procedure resulting in the desired form of the Lorentz transformations. Performing such a

\footnotetext{
${ }^{3}$ An eshaustive review of the interpretational problems of quantum mechanics can be found in $14,9,10,11,16$.
} 
program we have to distinguish, at least formally, one inertial frame - so called preferred frame. Thus, at least formally, the relativity principle may be broken. We discuss this question in the Section 2.1. Now, each inertial frame is determined by the fourvelocity of this frame with respect to the distinguished one. We denote fourvelocity of the preferred frame as seen by an inertial observer by $u^{\mu}$. Hereafter, quantities in the Einstein-Poincaré (EP) synchronization are denoted by the subscript (or superscript) E. Quantities in the CT synchronization will have no index. We use the natural units $(\hbar=c=1)$. According to [18, 19] transformation law beetwen inertial frames is determined by the following requirements:

$1^{0}$ the transformation group is isomorphic to the Lorentz group;

$2^{0}$ the average value of the light speed over closed paths is constant and equal to 1 ;

$3^{0}$ transformations are linear with respect to the coordinates (affinity);

$4^{0}$ under rotations coordinates transform in a standard way (isotropy)

$$
\begin{aligned}
& x^{\prime 0}\left(u^{\prime}\right)=x^{0}(u), \\
& \vec{x}^{\prime}\left(u^{\prime}\right)=R \vec{x}(u),
\end{aligned}
$$

here $R$ is the rotation matrix;

$5^{0}$ the instant time hyperplane $x^{0}=$ const is an invariant notion.

Notice that $1^{0}-4^{0}$ are the standard requirements, while nonstandard is the last one $\left(5^{0}\right)$. Consequently the transformation of coordinates between inertial frames in this synchronization has the following form (for contravariant coordinates)

$$
x^{\prime}\left(u^{\prime}\right)=D(\Lambda, u) x(u),
$$

where $D(\Lambda, u)$ is a $\Lambda$ and $u$ dependent $4 \times 4$ matrix, $\Lambda$ - element of the Lorentz group and $u^{\mu}$ is the fourvelocity of the preferred frame with respect to considered frame, so (11) is accompanied by

$$
u^{\prime}=D(\Lambda, u) u .
$$

Matrices $D(\Lambda, u)$ fulfill the following group composition rule

$$
D\left(\Lambda_{2}, D\left(\Lambda_{1}, u\right) u\right) D\left(\Lambda_{1}, u\right)=D\left(\Lambda_{2} \Lambda_{1}, u\right)
$$

so

$$
D^{-1}(\Lambda, u)=D\left(\Lambda^{-1}, D(\Lambda, u) u\right), \quad D(I, u)=I .
$$

Let $T(u)$ be the intertwining matrix connecting coordinates in CT and EP synchronizations. It means that for every contravariant fourvector $A^{\mu}$ we have

$$
A^{\mu}=T(u)^{\mu}{ }_{\nu} A_{E}^{\nu} .
$$

Therefore $D(\Lambda, u)$ is of the following form

$$
D(\Lambda, u)=T\left(u^{\prime}\right) \Lambda T^{-1}(u) .
$$


One can find the explicit form of $T(u)$ ([19]), namely

$$
T(u)=\left(\begin{array}{c|c}
1 & -\vec{u}^{T} u^{0} \\
\hline 0 & I
\end{array}\right) .
$$

Consequently $D(\Lambda, u)$ is given for rotations $(R \in S O(3))$ by

$$
D(R, u)=\left(\begin{array}{l|l}
1 & 0 \\
\hline 0 & R
\end{array}\right)
$$

while for boosts by

$$
D(W, u)=\left(\begin{array}{c|c}
\frac{1}{W^{0}} & 0 \\
\hline-\vec{W} & I+\frac{\vec{W} \otimes \vec{W}^{T}}{\left(1+\sqrt{1+(\vec{W})^{2}}\right)}-u^{0} \vec{W} \otimes \vec{u}^{T}
\end{array}\right),
$$

where $W^{\mu}$ denotes the fourvelocity of the primed frame $O_{u^{\prime}}$ with respect to the frame $O_{u}$. Fourvelocity $W^{\mu}$ can be expressed by $u$ and $u^{\prime}$

$$
W^{0}=\frac{u^{0}}{u^{\prime 0}}, \quad \vec{W}=\frac{\left(u^{0}+u^{\prime 0}\right)\left(\vec{u}-\vec{u}^{\prime}\right)}{\left[1+u^{0} u^{\prime 0}\left(1+\vec{u} \vec{u}^{\prime}\right)\right]} .
$$

Instead of $W^{\mu}$ we can use also velocity $\vec{V}=\frac{\vec{W}}{W^{0}}$. The corresponding form of all given above formulas in such a parametrization can be found by means of

$$
\frac{1}{W^{0}}=\sqrt{\left(1+u^{0} \vec{u} \vec{V}\right)^{2}-(\vec{V})^{2}} .
$$

The explicit relationship beetwen coordinates in EP and CT is given by

$$
\begin{array}{ll}
x_{E}^{0}=x^{0}+u^{0} \vec{u} \vec{x}, & \vec{x}_{E}=\vec{x}, \\
u_{E}^{0}=\frac{1}{u^{0}}, & \vec{u}_{E}=\vec{u} .
\end{array}
$$

We see that only the time coordinate changes. Note also, that in the same space point we have $\Delta x_{E}^{0}=\Delta x^{0}$ so the time lapse is the same in both synchronizations.

One can easily see that the line element

$$
d s^{2}=g_{\mu \nu}(u) d x^{\mu} d x^{\nu},
$$

is invariant under the transformations (6) if

$$
g(u)=\left(T(u) \eta T^{T}(u)\right)^{-1}
$$

where $\eta$ is the Minkowski metric tensor $\eta=\operatorname{diag}(+,-,-,-)$. The explicit form of the covariant metric tensor reads

$$
\left[g_{\mu \nu}\right]=\left(\begin{array}{c|c}
1 & u^{0} \vec{u}^{T} \\
\hline u^{0} \vec{u} & -I+\left(u^{0}\right)^{2} \vec{u} \otimes \vec{u}^{T}
\end{array}\right),
$$


while the contravariant one has the form

$$
g^{-1}(u)=\left(\begin{array}{c|c}
\left(u^{0}\right)^{2} & u^{0} \vec{u}^{T} \\
\hline u^{0} \vec{u} & -I
\end{array}\right),
$$

so the space line element is the Euclidean one: $d l^{2}=d \vec{x}^{2}$. Let us notice here that the triangular form of the boost matrix (9) implies, that under the Lorentz transformations the time coordinate is only rescaled by a positive factor $\left(x^{\prime 0}=\right.$ $\left.\frac{1}{W^{0}} x^{0}\right)$; the space coordinates do not mix to it. One can also easily check that the following, very useful, relations hold

$$
\underline{u}=\underline{0} \quad \text { and } \quad \frac{1}{\left(u^{0}\right)^{2}}-(\vec{u})^{2}=1 .
$$

Heareafter the three vector part of a covariant (contravariant) fourvector $a_{\mu}$ $\left(a^{\mu}\right)$ will be denoted by $a(\vec{a})$ respectively.

\subsection{Geometric description of the CT synchronization}

We can also provide a geometric description of the special relativity in the CT synchronization scheme in the language of frame bundles. To do this, let us denote:

$M$ - the Minkowski space-time,

$L_{+}^{\uparrow}$ - ortochronous Lorentz group (the group of space-time transformations);

$F\left(L_{+}^{\uparrow}\right)$ - the set of all frames in the space $M$ obtained by action of $L_{+}^{\uparrow}$ on one particular (but arbitrary) frame in the space $M$; thus $F\left(L_{+}^{\uparrow}\right)$ is isomorphic to the group $L_{+}^{\uparrow}$. An element of $F\left(L_{+}^{\uparrow}\right)$ corresponding, by means of this isomorphism, to the element $g \in L_{+}^{\uparrow}$ is designated by $e(g)$.

Now let us consider the following structure

$$
M_{w}=\left[L_{+}^{\uparrow},\left(F\left(L_{+}^{\uparrow}\right) \times M, M, p r_{2}\right), \pi_{w}, \psi_{w}\right],
$$

where $\mathrm{pr}_{2}$ is the canonical projection on the second factor of the cartesian product; therefore $\left(F\left(L_{+}^{\uparrow}\right) \times M, M, p r_{2}\right)$ is a frame bundle with the typical fibre $F\left(L_{+}^{\uparrow}\right) \cdot \pi_{w}$ is a projection on a fixed time-like fourvector $w$, while $\psi_{w}$ is the action of the group $L_{+}^{\uparrow}$ on the bundle $\left(F\left(L_{+}^{\uparrow}\right) \times M, M, p r_{2}\right)$ fulfilling the following conditions:

$$
\begin{gathered}
\left(e^{\prime}(g), x^{\prime}\right)=(e(k g), x), \\
e^{\mu}(k g, x)=D(k, g)^{\mu}{ }_{\nu} e^{\nu}(g, x), \\
D^{T-1}(k, g) \pi_{w} D^{-1}(k, g)=\pi_{w} .
\end{gathered}
$$

where $k \in L_{+}^{\uparrow}, x \in M$. It is clear that we consider here Lorentz transformations as passive transformations - the action of the Lorentz group changes the 
observer, not the physical state. In our language it means that the action of the Lorentz group changes the frame $e(g)$. The condition (19) means that the action $\psi_{w}$ is trivial on the manifold $M$; the group $L_{+}^{\uparrow}$ acts only in the fiber. The second condition (20) says that the action $\psi_{w}$ is linear on frames. Now, we associate the time direction with $w$ which means that the projector $\pi_{w}=\frac{w \otimes w}{w^{2}}$ is equal to $\pi_{e^{0}}$

$$
\pi_{e^{0}}=\pi_{w},
$$

so, after this identification, $\pi_{w}$ in the $e^{\mu}$ basis $\left(\pi_{w}=\left(\pi_{w}\right)_{\mu \nu} e^{\mu} \otimes e^{\nu}\right)$ reads

$$
\left[\left(\pi_{e^{0}}\right)_{\mu \nu}\right]=\left[\left(\pi_{w}\right)_{\mu \nu}\right]=\left(\begin{array}{cccc}
1 & 0 & 0 & 0 \\
0 & 0 & 0 & 0 \\
0 & 0 & 0 & 0 \\
0 & 0 & 0 & 0
\end{array}\right)
$$

This construction defines a time-orientation of $M$ along $w$. Now the matrix $D(k, g)$ can be expressed by $D(\Lambda, u)$ given in eqs. (89) as follows: let $\Lambda_{1}=k$, $\Lambda_{2}=g$, then

$$
D(k, g)=D\left(\Lambda_{1}, \Lambda_{2} \tilde{u}\right),
$$

and $\tilde{u}=(1, \overrightarrow{0})$. The last condition (21) means that the direction of the fourvector $w$ is invariant under the action of the group $L_{+}^{\uparrow}$.

Thus we have a collection of time-oriented space-times $M_{w}$, where $w$ is the arbitrary time-like fourvector. The objects $M_{w}$ and $M_{w^{\prime}}$ corresponding to different $w$ and $w^{\prime}$ are evidently connected by the action of another Lorentz group $L_{+}^{\uparrow(S)}$ (the so called synchronization group - see [19]). The whole family of time-oriented space-times $M_{w}$ together with the transformations $\varphi$ of the synchronization group, treated as morphisms, form a category

$$
\mathcal{A}=\left(M_{w}, \varphi\right) .
$$

The action $\varphi$ of the synchronization group $L_{+}^{\uparrow(S)}$ is defined in the most natural way

$$
\varphi\left(M_{w}\right)=M_{\Lambda^{S} \text { ow }}, \quad \Lambda^{S} \in L_{+}^{\uparrow(S)} .
$$

From the physical point of view all choices of the element of the category $\mathcal{A}$ are equivalent provided that the relativity principle holds. However, if we want to introduce covariant canonical formalism for a relativistic free particle on the classical level or to define a proper position operator for such a particle on the quantum level we have to give up the relativity principle; a consistent description is possible only if we use a fixed element of the category $\mathcal{A}$. In this case also causal problems connected with breaking of Bell's inequalities in QM disappear. Summarizing, formulation of special relativity in terms of the category $\mathcal{A}$ is equivalent to the standard one, however, whenever the notion of localizability or absolute causality are incorporated, the group of morphisms is broken i.e. a concrete time orientation is fixed. The advantage of the use of 
$\mathcal{A}$ (family of CT synchronizations) in comparison with $\mathrm{EP}$ scheme is, that in the former case we can consistently define the position operator while in the later one it is impossible. Moreover this construction shows, that some notions (like localizability) are simultaneously compatible with quantum mechanics and Poincaré covariance only if we resign with democracy between inertial frames, i.e. if a privileged frame is distinguished.

\section{Canonical formalism in the CT synchroniza- tion}

For a relativistic free particle we postulate the following action functional

$$
S_{12}=-m \int_{\lambda_{1}}^{\lambda_{2}} \sqrt{d s^{2}},
$$

where $d s^{2}=g_{\mu \nu}(u) \frac{d x^{\mu}}{d \lambda} \frac{d x^{\nu}}{\lambda} d \lambda^{2}$ and $\lambda$ is a trajectory parameter. We define the fourvelocity in the standard way: $\omega^{\mu}=\frac{d x^{\mu}}{d \lambda}=\dot{x}^{\mu}$. Then the velocity has the form: $\vec{v}=\frac{d \vec{x}}{d x^{0}}=\frac{\vec{\omega}}{\omega^{0}}$. Choosing the parameter $\lambda$ as the lenght of the trajectory, $d \lambda=\sqrt{d s^{2}}$, we obtain the following condition

$$
\omega^{2}=g_{\mu \nu}(u) \omega^{\mu}(u) \omega^{\nu}(u)=1,
$$

which implies

$$
\dot{\omega}^{\mu}=\ddot{x}^{\mu}=0 .
$$

Using the formula (15) we can derive from (27) the Lagrangian

$$
L=-m \sqrt{\left(1+u^{0} \vec{u} \vec{v}\right)^{2}-(\vec{v})^{2}} .
$$

Now we can calculate the canonical momenta

$$
\pi_{i}=\frac{\partial L}{\partial v^{i}}=\frac{m\left[v^{i}-u^{i} u^{0}\left(1+u^{0} \vec{u} \vec{v}\right)\right]}{\sqrt{\left(1+u^{0} \vec{u} \vec{v}\right)^{2}-(\vec{v})^{2}}}=-m \omega_{i},
$$

and the Hamiltonian

$$
H=\pi_{k} v^{k}-L=\frac{1}{u^{0}}\left(\vec{u} \underset{\rightarrow}{\pi}+\sqrt{(\vec{u} \pi)^{2}+(\underset{\sim}{\pi})^{2}+m^{2}}\right)=m \omega_{0},
$$

where $\underset{\rightarrow}{\pi}=\left(\pi_{1}, \pi_{2}, \pi_{3}\right)$. So the covariant fourmomentum can be defined by

$$
k_{\mu}=m \omega_{\mu} .
$$

It is easy to check, that $k_{\mu}$ fulfills the following dispersion relation

$$
k^{2}=g^{\mu \nu}(u) k_{\mu} k_{\nu}=m^{2} .
$$


Now we introduce the Poisson bracket

$$
\{A, B\}=-\left(\delta^{\mu}{ }_{\nu}-\frac{k^{\mu} u_{\nu}}{u k}\right)\left(\frac{\partial A}{\partial x^{\mu}} \frac{\partial B}{\partial k_{\nu}}-\frac{\partial B}{\partial x^{\mu}} \frac{\partial A}{\partial k_{\nu}}\right),
$$

where all variables $x^{\mu}, k_{\nu}$ are treated as independent; in particular $k_{0}$ is not $a$ priori connected with $k_{i}$ via the disperssion relation (34). The Poisson bracket defined by the above formula satisfies all necessary conditions:

- it is linear with respect to both factors, antisymmetric, satisfies the Leibniz rule and fulfills the Jacobi identity;

- it is manifestly Poincaré covariant in the CT synchronization;

- it is consistent with the constraint (34), i.e. $\left\{k^{2}, k_{\nu}\right\}=\left\{k^{2}, x^{\mu}\right\}=0$; therefore there is no reason to introduce a Dirac bracket.

- it is consistent with the Hamilton equations (37);

In particular from (35) we get

$$
\begin{array}{ll}
\left\{x^{\mu}, x^{\nu}\right\}=0, & \left\{x^{0}, k_{\mu}\right\}=0, \\
\left\{x^{i}, k_{j}\right\}=-\delta_{j}^{i}, & \left\{x^{i}, k_{0}\right\}=\frac{k^{i}}{k^{0}}, \\
\left\{k_{\mu}, k_{\nu}\right\}=0 . &
\end{array}
$$

The Hamilton equations for a free particle have the desirable form

$$
\frac{d x^{i}}{d t}=\frac{\partial H}{\partial \pi_{i}}=\frac{k^{i}}{k^{0}}=v^{i}, \quad \frac{d k_{i}}{d t}=-\frac{\partial H}{\partial x^{i}}=0,
$$

where $H$ is given by (32). In general, the equation of motion for an observable $\Omega\left(x^{\mu}, k_{\nu}\right)$ expressed by the Poisson bracket $(35)$ is

$$
\frac{d \Omega}{d t}=\frac{\partial \Omega}{\partial t}+\left\{\Omega, k_{0}\right\}
$$

In the above equation as well as in (35) $k_{0}$ is treated as an independent variable. Solution of (38) can be subduced to the constraint surface (34).

\section{Quantum theory in the CT synchronization}

The results of previous sections imply that in the CT synchronization we have absolute causality so in this approach disappear all causal problems connected with violation of Bell inequalities. Quantum theory remains non-local but it is causal. In our approach we are able, in analogy to the classical Poisson algebra described in section 3, to introduce a Poincaré covariant algebra of momentum and position operators satisfying all fundamental physical requirements. This is done in the section 4.2. Properties of the introduced position operator will be discussed in details in the sections 6.2 and 6.3 . 


\subsection{Preliminaries}

In the CT synchronization the following point of view is the most natural one: with each inertial observer $O_{u}$ we connect his own Hilbert space $H_{u}$ (space of states). The states vectors from $H_{u}$ are denoted by $u$ : $|u, \ldots\rangle$. In other words we have a bundle of Hilbert spaces corresponding to the bundle of frames described in sec. 2.1. In such an interpretation we have to distinguish carefuly active and passive transformations, because in our approach active transformations are represented by operators acting in one Hilbert space while passive ones by operators acting beetwen different Hilbert spaces. So, in particular, the Lorentz group transformations are considered as passive ones. Now, let $U(\Lambda)$ be an operator representing a Lorentz group element $\Lambda$. We postulate the following, standard, transformation law for a contravariant fourvector operator

$$
U(\Lambda) \hat{A}(u)^{\mu} U^{-1}(\Lambda)=\left(D^{-1}(\Lambda, u)\right)_{\nu}^{\mu} \hat{A}\left(u^{\prime}\right)^{\nu}
$$

where $D(\Lambda, u)$ is given by eqs. (8.9) and $u^{\prime}=D(\Lambda, u) u$; for a covariant fourvector $\hat{A}(u)_{\mu}$ we have to replace $D^{-1}$ by $D^{T}$ on the right hand side of $(39)$. Let $\Omega$ be a fourvector observable. In the space $H_{u}$ the observable $\Omega$ is represented by an operator $\hat{\Omega}^{\mu}(u)$. Now let two inertial observers $O_{u}$ and $O_{u^{\prime}}$ measure independently the value of the observable $\Omega$ for a physical system being in the same physical state ${ }^{4}$. Let this state in the space $H_{u}$ be described by the eigenvector $|\omega, u, \ldots\rangle$ of the $\Omega^{\mu}$. Then in the space $H_{u^{\prime}}$ the same state is described by the vector

$$
\left|\omega^{\prime}, u^{\prime}, \ldots\right\rangle=U(\Lambda)|\omega, u, \ldots\rangle,
$$

where $u^{\prime}=D(\Lambda, u) u, \omega^{\prime}=D(\Lambda, u) \omega$. As a result of measurement of $\Omega$ the observer $O_{u}$ will receive the value $\omega$

$$
\hat{\Omega}^{\mu}(u)|\omega, u, \ldots\rangle=\omega^{\mu}|\omega, u, \ldots\rangle .
$$

As a result of measurment the observer $O_{u^{\prime}}$ should obtain the value $\omega^{\prime}=$ $D(\Lambda, u) \omega$. So in the space of states $H_{u^{\prime}}$ the observable $\Omega$ is represented by an operator $\hat{\Omega}^{\mu}\left(u^{\prime}\right)$, because

$$
\begin{aligned}
& \hat{\Omega}\left(u^{\prime}\right)\left|\omega^{\prime}, u^{\prime}, \ldots\right\rangle=U(\Lambda) D(\Lambda, u) \hat{\Omega}(u)|\omega, u, \ldots\rangle= \\
& \quad=U(\Lambda) D(\Lambda, u) \omega(u)|\omega, u, \ldots\rangle= \\
& \quad=\omega^{\prime}\left(u^{\prime}\right)\left|\omega^{\prime}, u^{\prime}, \ldots\right\rangle,
\end{aligned}
$$

where we have used (39,40). To conclude this section we provide the interpretation of the operator $\hat{\Omega}^{\prime}\left(u^{\prime}\right)=D(\Lambda, u) \hat{\Omega}(u)$. We have

$$
\begin{aligned}
& \hat{\Omega}^{\prime \mu}\left(u^{\prime}\right)|\omega, u, \ldots\rangle=D_{\nu}^{\mu}(\Lambda, u) \hat{\Omega}^{\nu}(u)|\omega, u, \ldots\rangle= \\
& \quad=D_{\nu}^{\mu}(\Lambda, u) \omega^{\nu}(u)|\omega, u, \ldots\rangle=\omega^{\prime \mu}\left(u^{\prime}\right)|\omega, u, \ldots\rangle .
\end{aligned}
$$

\footnotetext{
${ }^{4}$ Of course, we should imagine an ansamble of identical copies of a physical system in the same prepared state.
} 
Thus $\hat{\Omega}^{\prime}\left(u^{\prime}\right)$ is an operator when acting on a vector describing the state of a physical system in the space of the observer $O_{u}$ gives the same result as seen by the observer $O_{u^{\prime}}$ performing measurement on this system being in the same physical state.

\subsection{Algebra of momenta and positions}

We can introduce in each space $H_{u}$ the Hermitean fourmomentum operators $\hat{p}_{\lambda}(u)$ (generators of translations). These operators are interpreted as observables in the corresponding reference frame. In the CT synchronization we can go further and introduce in each space $H_{u}$ Hermitean position operators $\hat{x}^{\mu}(u)$. According to the Poisson bracket on the classical level (35) we postulate the following commutators between $\hat{x}^{\mu}(u)$ and $\hat{p}_{\lambda}(u)$

$$
\begin{gathered}
{\left[\hat{x}^{\mu}(u), \hat{p}_{\lambda}(u)\right]=i\left(\frac{u_{\lambda} \hat{p}^{\mu}(u)}{u \hat{p}(u)}-\delta_{\lambda}^{\mu}\right),} \\
{\left[\hat{p}_{\mu}(u), \hat{p}_{\nu}(u)\right]=0} \\
{\left[\hat{x}^{\mu}(u), \hat{x}^{\nu}(u)\right]=0 .}
\end{gathered}
$$

In particular

$$
\begin{gathered}
{\left[\hat{x}^{0}(u), \hat{p}_{\lambda}(u)\right]=0,} \\
{\left[\hat{x}^{i}(u), \hat{p}_{j}(u)\right]=-i \delta^{i}{ }_{j},} \\
{\left[\hat{x}^{i}(u), \hat{p}_{0}(u)\right]=i \frac{\hat{p}^{i}(u)}{\hat{p}^{0}(u)} .}
\end{gathered}
$$

We see that $\hat{x}^{0}$ commutes with all observables, it allows us to interprete $\hat{x}^{0}$ as a parameter just like in the standard nonrelativistic quantum mechanics. We have to stress here once again that above commutation relations defining position operators are covariant in the CT synchronization. It can be checked directly; one simply has to use the eqs. (8,9,39) and to transform the eqs. (44, 15, 46) to another reference frame. One can also check that

$$
\left[\hat{x}^{\mu}(u), \hat{p}^{2}\right]=0,
$$

which means that localized states have definite mass.

\section{$5 \quad$ Unitary orbits of the Poincaré group for $k^{2}>0$ and spin operators}

According to our interpretation we deal with a bundle of Hilbert spaces $H_{u}$ rather than with a single space of states. Therefore transformations of the Lorentz group induce an orbit in this bundle. In this section we construct and 
classify unitary orbits of the Poincare group in the above mentioned bundle of Hilbert spaces. As we will see, the unitary orbits are classified with help of mass and spin, similarly as for the standard unitary representations of the Poincaré group.

\subsection{Unitary orbits}

As in the standard case we assume that the eigenvectors $|k, u, \ldots\rangle$ of the fourmomentum operators

$$
\hat{p}_{\mu}(u)|k, u, \ldots\rangle=k_{\mu}|k, u, \ldots\rangle
$$

with $k^{2}=m^{2}$, form a base in the Hilbert space $H_{u}$. We adopt the following Lorentz-covariant normalization

$$
\left\langle k^{\prime}, u, \ldots \mid k, u, \ldots\right\rangle=2 k^{0} \delta^{3}\left(\underline{k}^{\prime}-\underset{\rightarrow}{k}\right),
$$

where $k$ denotes the space part of the covariant fourvector $k_{\mu}$ and $k^{0}=g^{0 \mu} k_{\mu}$ is positive. Energy $k_{0}$ is the solution of the dispersion relation $k^{2}=m^{2}$ and is given by

$$
k_{0}=\frac{1}{u^{0}}\left(-\vec{u} \underset{\rightarrow}{k}+\sqrt{(\vec{u} \underset{\rightarrow}{k})^{2}+(\underset{\rightarrow}{k})^{2}+m^{2}}\right)
$$

so

$$
k^{0} \equiv \omega(\underline{k})=u^{0} \sqrt{(\vec{u} \underline{\underline{s}})^{2}+(\underline{k})^{2}+m^{2}} .
$$

In the construction of the unitary irreducible orbits we use the operator $e^{-i q} \hat{\vec{x}}(u)$. Action of this operator on the basis states can be determined by using its unitarity, normalization of the basis vectors (52) and the commutation relations (44). Its final form is

$$
e^{-i q \overrightarrow{\vec{x}}(u)}|k, u, \ldots\rangle=e^{i q_{0} \hat{x}^{0}(u)} \sqrt{\frac{u k}{u(k+q)}}|k+q, u, \ldots\rangle,
$$

where on the right hand side of (55) $q_{0}$ is determined by $\underset{\rightarrow}{k}, q$ and $u$; namely

$$
\begin{aligned}
q_{0}= & \frac{1}{u^{0}}\left(-\vec{u} \underline{q}-\sqrt{(\vec{u} k)^{2}+(\underline{k})^{2}+m^{2}}+\right. \\
& \left.+\sqrt{(\vec{u} \underline{q}+\vec{u} k \underline{s})^{2}+(\underline{k}+\underline{q})^{2}+m^{2}}\right) .
\end{aligned}
$$

The basis vectors of the space $H_{u}$ can be generated from a vector representing a particle at rest with respect to the preferred frame. Firstly we act $U\left(L_{u}\right)$ on such

\footnotetext{
${ }^{5}$ There is a freedom in choosing the phase factor on the right hand side of (55); it is determined here by the requirement that no phase factor on the right hand side of (60) appears.
} 
a vector; the resulting state has fourmomentum $m u_{\mu}$ and belongs to $H_{u}$. Next, by means of the formula (55), we generate in $H_{u}$ a vector with fourmomentum $k_{\mu}$. Precisely

$$
|k, u, \ldots\rangle=\sqrt{\frac{u k}{m}} e^{-i\left(k_{\mu}-m u_{\mu}\right) \hat{x}^{\mu}(u)} U\left(L_{u}\right)|\underset{\sim}{k}, \tilde{u}, \ldots\rangle,
$$

where:

$$
\tilde{u}=(1, \overrightarrow{0}), \quad \underset{\sim}{k}=(m, \underset{\sim}{0}), \quad u=D\left(L_{u}, \tilde{u}\right) \tilde{u} .
$$

The above mentioned orbit induced by the action of the operator $U(\Lambda)$ in the bundle of Hilbert spaces is fixed by the following covariant conditions

$-k^{2}=m^{2}$

- $\varepsilon\left(k^{0}\right)=i n v$., for physical representations $k^{0}>0, \varepsilon\left(k^{0}\right)=1$.

As a consequence there exists a positive defined, Lorentz invariant measure

$$
d \mu(k, m)=d^{4} k \theta\left(k^{0}\right) \delta\left(k^{2}-m^{2}\right)
$$

Now, applying the Wigner method and using eq. (39) one can easily determine the action of the operator $U(\Lambda)$ on the basis vector. We find

$$
U(\Lambda)|k, u, m ; s, \sigma\rangle=\mathcal{D}_{\sigma \lambda}^{s}{ }^{-1}\left(R_{\Lambda, u}\right)\left|k^{\prime}, u^{\prime}, m ; s, \lambda\right\rangle,
$$

where

$$
\begin{gathered}
u^{\prime}=D(\Lambda, u) u=D\left(L_{u^{\prime}}, \tilde{u}\right) \tilde{u} \\
k^{\prime}=D^{T-1}(\Lambda, u) k \\
R_{\Lambda, u}=D\left(R_{\Lambda, u}, \tilde{u}\right)=D^{-1}\left(L_{u^{\prime}}, \tilde{u}\right) D(\Lambda, u) D\left(L_{u}, \tilde{u}\right) \subset S O(3)
\end{gathered}
$$

and $\mathcal{D}_{\sigma \lambda}^{s}\left(R_{\Lambda, u}\right)$ is the standard spin $s$ rotation matrix $s=0, \frac{1}{2}, 1, \ldots ; \sigma, \lambda=$ $-s,-s+1, \ldots, s-1, s . D\left(R_{\Lambda, u}, \tilde{u}\right)$ is the Wigner rotation belonging to the little group of a vector $\tilde{u}$. Let us stress that in our approach, contrary to the standard one, representations of the Poincaré group are induced from the little group of a vector $\tilde{u}$, not $\underset{\sim}{k}$. Finally, the normalization (52) takes the form

$$
\left\langle k, u, m ; s, \lambda \mid k^{\prime}, u, m ; s^{\prime}, \lambda^{\prime}\right\rangle=2 k^{0} \delta^{3}\left(\underline{k}^{\prime}-\underline{k}\right) \delta_{s^{\prime} s} \delta_{\lambda^{\prime} \lambda} .
$$

\subsection{Spin}

Now we describe in some details transformation properties of a second rank covariant tensor operator. These results are used in the discussion of the spin. Let $\hat{M}(u)=\left[\hat{M}_{\mu \nu}(u)\right]$ be a tensor operator. The transformation law for this tensor can be deduced from (39) and can be written in the matrix notation as

$$
U(\Lambda) \hat{M}(u) U^{-1}(\Lambda)=D^{T}(\Lambda, u) \hat{M}\left(u^{\prime}\right) D(\Lambda, u) .
$$


The lower-triangular form of the matrix $D(\Lambda, u)$ (see eq. (8) and (9)) implies that the space part of $\hat{M}$ transform into itself, namely

$$
U(\Lambda) \hat{M}_{i j}(u) U^{-1}(\Lambda)=\Omega_{k i}(\Lambda, u) \hat{M}_{k l}\left(u^{\prime}\right) \Omega_{l j}(\Lambda, u),
$$

where $\Omega(\Lambda, u)$ denotes the space part of the matrix $D(\Lambda, u)$. By means of the triangular form of $D(\Lambda, u)$ it is easy to see that

$$
g_{i j}\left(u^{\prime}\right)=\Omega_{k i}^{-1}(\Lambda, u) g_{k l}(u) \Omega_{l j}^{-1}(\Lambda, u),
$$

where $g_{i j}$ are the space components of the covariant metric tensor $g_{\mu \nu}$. Therefore, denoting by $\gamma_{i j}$ the inverse of the matrix of the space part of the $g_{\mu \nu}(u)$, that is

$$
\gamma_{i j}(u)=\left[g_{i j}\right]^{-1}=-\left(\delta_{i j}+u^{i} u^{j}\right)
$$

one can easily show that the bilinear form

$$
\hat{M}^{2}=\gamma_{i k}(u) \gamma_{j l}(u) \hat{M}_{i j}(u) \hat{M}_{k l}(u)
$$

is a Poincaré invariant operator. Let us introduce the spin operators $\hat{S}_{i j}(u)$ transforming covariantly according to (66) such that

$$
U(\Lambda) \hat{S}_{i j}(u) U^{-1}(\Lambda)=\Omega_{k i}(\Lambda, u) \hat{S}_{k l}\left(u^{\prime}\right) \Omega_{l j}(\Lambda, u),
$$

and defined by the action on the basis vectors

$$
\hat{S}_{i j}(u)|k, u, m ; s, \lambda\rangle=-\mathcal{S}_{i j}^{s}(u)_{\lambda \sigma}|k, u, m ; s, \sigma\rangle .
$$

By means of the equation (60), the eqs. (70) and (71) imply the following consistency condition

$$
\mathcal{D}^{s}\left(R_{\Lambda, u}\right) \mathcal{S}_{i j}^{s}(u) \mathcal{D}^{s}\left(R_{\Lambda, u}^{-1}\right)=\Omega_{k i}(\Lambda, u) \mathcal{S}_{k l}^{s}\left(u^{\prime}\right) \Omega_{l j}(\Lambda, u) .
$$

Therefore, using the fact that $R_{L_{u}, \tilde{u}}=I$ and

$$
D\left(L_{u}, \widetilde{u}\right)=\left(\begin{array}{c|c}
u^{0} & 0 \\
\hline \vec{u} & I+\frac{u^{0}}{1+u^{0}} \vec{u} \otimes \vec{u}^{T}
\end{array}\right)
$$

$\left(\right.$ so $\left.\Omega_{i j}\left(L_{u}, \tilde{u}\right)=\delta_{i j}+\frac{u^{0}}{1+u^{0}} u^{i} u^{j}\right)$, one obtains

$$
\mathcal{S}_{i j}^{s}(u)=\tilde{\mathcal{S}}_{i j}^{s}+\frac{\left(u^{0}\right)^{2}}{1+u^{0}}\left(u^{j} \delta_{l i}-u^{i} \delta_{l j}\right) u^{k} \tilde{\mathcal{S}}_{k l}^{s},
$$

where $\tilde{\mathcal{S}}_{i j}^{s}:=\mathcal{S}_{i j}^{s}(\tilde{u})$ are assumed to be Hermitean matrix generators of the unitary representation $\mathcal{D}^{s}(R)$ of $S O(3)$, i.e.

$$
\tilde{\mathcal{S}}_{i j}^{s}=-\tilde{\mathcal{S}}_{j i}^{s}=\left(\tilde{\mathcal{S}}_{i j}^{s}\right)^{\dagger}
$$


and

$$
\left[\tilde{\mathcal{S}}_{i j}^{s}, \tilde{\mathcal{S}}_{k l}^{s}\right]=-i\left(\delta_{i l} \tilde{\mathcal{S}}_{j k}^{s}+\delta_{j k} \tilde{\mathcal{S}}_{i l}^{s}-\delta_{i k} \tilde{\mathcal{S}}_{j l}^{s}-\delta_{j l} \tilde{\mathcal{S}}_{i k}^{s}\right) .
$$

Consequently, in an arbitrary frame

$$
\mathcal{S}_{i j}^{s}(u)=-\mathcal{S}_{j i}^{s}(u)=\mathcal{S}_{i j}^{s \dagger}(u),
$$

and

$$
\begin{aligned}
& {\left[\mathcal{S}_{i j}^{s}(u), \mathcal{S}_{k l}^{s}(u)\right]=} \\
& \quad=i\left(g_{i l}(u) \mathcal{S}_{j k}^{s}(u)+g_{j k}(u) \mathcal{S}_{i l}^{s}(u)-g_{i k}(u) \mathcal{S}_{j l}^{s}(u)-g_{j l}(u) \mathcal{S}_{i k}^{s}(u)\right) .
\end{aligned}
$$

Therefore, the spin operators $\hat{S}_{i j}(u)=-\hat{S}_{j i}(u)$ are Hermitean and satisfy the same algebra

$$
\begin{aligned}
& {\left[\hat{S}_{i j}(u), \hat{S}_{k l}(u)\right]=} \\
& \quad=i\left(g_{i l}(u) \hat{S}_{j k}(u)+g_{j k}(u) \hat{S}_{i l}(u)-g_{i k}(u) \hat{S}_{j l}(u)-g_{j l}(u) \hat{S}_{i k}(u)\right) .
\end{aligned}
$$

Now, according to $(69)$ one can define the invariant spin square operator

$$
\begin{aligned}
\hat{S}^{2} & =\frac{1}{2} \gamma_{i k}(u) \gamma_{j l}(u) \hat{S}_{i j}(u) \hat{S}_{k l}(u)= \\
& =\frac{1}{2} \hat{S}_{i j}(u) \hat{S}_{i j}(u)+u^{i} u^{j} \hat{S}_{i k}(u) \hat{S}_{j k}(u) .
\end{aligned}
$$

Consequently

$$
\hat{S}^{2}|k, u, m ; s, \lambda\rangle=s(s+1)|k, u, m ; s, \lambda\rangle .
$$

Finally, as follows from (71), (51) and (55), $\hat{S}_{i j}(u)$ commute with $\hat{p}_{\mu}(u)$ and $\hat{x}^{\mu}(u)$ i.e.

$$
\left[\hat{S}_{i j}(u), \hat{p}_{\mu}(u)\right]=\left[\hat{S}_{i j}(u), \hat{x}^{\mu}(u)\right]=0 .
$$

Summarizing, the introduced covariant spin operator has properties showing its advantage in comparison with the standard one. In particular the algebra generated by $\hat{p}_{\mu}(u), \hat{x}^{\mu}(u)$ and $\hat{S}_{i j}(u)$ - the equations $\left.44,45,46,79,82\right)$ - is evidently covariant under the Poincaré group action.

\section{The position operator and localized states}

In the section 6.1 we recall briefly the Newton-Wigner position operator. The section 6.2 is devoted to localized states and derivation of a functional form of the introduced in the section 1 position operator. Section 6.3 is devoted to description of localized states and position operator in the Hilbert space with a fully invariant measure, resembling the nonrelativistic one. 


\subsection{The Newton-Wigner operator}

In the non-relativistic quantum mechanics the situation is clear, we can define the position operator which fulfills all the conditions stated in the introduction (covariance is of course understood with respect to the Galilei group). Its construction and properties are very well known and we do not intent to describe them in this section. In the relativistic case situation is much more complicated. One of the earliest definitions of the position operator is due to Newton and Wigner 14. In this approach the authors try first to find states of the particle localized at a given point $(t, \vec{a})$ and then to write down the corresponding position operators. Let $S_{\vec{a}}$, the set of states $\psi_{\vec{a}, 0}$ localized at $\vec{a} \in \mathbb{R}^{3}$ at $t=0$, be the subset of the Hilbert space $\mathcal{H}$ of the unitary irreducible representation of the universal covering group of the Poincaré group. The Newton-Wigner postulates are as follows:

- the set $S_{\vec{a}}$ is a linear subspace of $\mathcal{H}$;

- $S_{\vec{a}}$ is invariant under rotations around point $\vec{a}$, reflections in $\vec{a}$, and time inversions;

- $S_{\vec{a}}$ is orthogonal to all its space translates, i.e. under the space translations each $\psi_{\vec{a}, 0} \in S_{\vec{a}}$ transforms to a state from $\mathcal{H}$ which is orthogonal to all states from $S_{\vec{a}}$;

- certain regularity conditions.

As an example let us discuss shortly the Newton-Wigner position operator for a spinless particle. In this case $\mathcal{H}$ is a linear space of solutions to the KleinGordon equation with positive energy. Using the Fourier transform one can obtain the states localized at $\vec{a} \in \mathbb{R}^{3}$ at $t=0$ in the momentum representation, namely

$$
S_{\vec{a}}=\left\{\psi_{\vec{a}, 0}(k)=\frac{1}{(2 \pi)^{3 / 2}} k_{0}^{1 / 2} e^{-i \vec{k} \vec{a}}\right\} .
$$

The corresponding position operators are given by:

$$
\hat{q}^{k}=-i\left(\frac{\partial}{\partial k_{i}}+\frac{1}{2} \frac{k^{i}}{(\vec{k})^{2}+m^{2}}\right) .
$$

The main results obtained by Newton and Wigner may be summarized in the following way

- position operator exists for massive particles with arbitrary spin,

- it is hermitean,

- it has commuting components,

- under rotations transforms like a vector,

- $\left[\hat{q}^{k}, \hat{k}_{i}\right]=-i \delta_{i}^{k}$ (canonical commutation relations with momentum operators),

- it is not covariant,

- localized states are not covariant,

- massles particles with spin are not localizable. 
Of course a lot of trials have been undertaken to remove all the unsatisfactory features of the Newton-Wigner approach, but to the best knowledge of the authors no one of them has been fully succesfull. For a review see 4,15 .

\subsection{Localized states and momentum representation of the position operator}

In this section we briefly describe some properties of the introduced in the section 4.2 position operator. Firstly let us find localized states in the Schrödinger picture. Taking into account the eqs. (50) and (82) we find that $\hat{x}^{\mu}(u)$ commutes with $\hat{p}^{2}$ and $\hat{S}_{i j}(u)$ so all these three operators have common eigenvectors and consequently localized states have definite mass and spin. Let $|\vec{\xi}, \tau, u, m ; s, \lambda\rangle$ denotes a state localized at the time $\tau$ in the space point $\vec{\xi}$

$$
\hat{\vec{x}}(u)|\vec{\xi}, \tau, u, m ; s, \lambda\rangle=\vec{\xi}|\xi, \tau, u, m ; s, \lambda\rangle .
$$

The state $|\vec{\xi}, \tau, u, m ; s, \lambda\rangle$ can be expressed with help of the invariant measure (59) in terms of the basis vectors $|k, u, m ; s, \lambda\rangle$, namely

$$
|\vec{\xi}, \tau, u, m ; s, \lambda\rangle=\frac{1}{(2 \pi)^{3 / 2}} \int \frac{d^{3} \underline{\underline{k}}}{2 \omega(\underline{\underline{k}})} \sqrt{u k} e^{i k} \vec{\xi}|k, u, m ; s, \lambda\rangle .
$$

Now, after an arbitrary time $t$ this state evolves to

$$
|\vec{\xi}, \tau, u, m ; s, \lambda ; t\rangle=\frac{1}{(2 \pi)^{3 / 2}} \int \frac{d^{3} k}{2 \omega(\underline{k})} \sqrt{u k} e^{i k_{\mu} \xi^{\mu}}|k, u, m ; s, \lambda\rangle
$$

with $\xi^{0}=\tau-t$. One can easily check that these states are normalized as follows

$$
\left\langle\vec{\xi}^{\prime}, \tau, u, m ; s^{\prime}, \lambda^{\prime} ; t \mid \vec{\xi}, \tau, u, m ; s, \lambda ; t\right\rangle=\frac{1}{2 u^{0}} \delta^{3}\left(\vec{\xi}-\vec{\xi}^{\prime}\right) \delta_{s s^{\prime}} \delta_{\lambda \lambda^{\prime}}
$$

It is worthwhile to notice here that the states given by the eq. (86) are covariant in the CT synchronization, i.e. a state localized in the time $t=\tau$ for the observer $O_{u}$ is localized in the time $t^{\prime}=\tau^{\prime}=D_{0}^{0}(\Lambda, u) \tau$ for the observer $O_{u}$ too. Let us discuss a realization of the position operator in the momentum representation. Wave functions in momentum representation are defined in the standard way

$$
\psi_{\lambda}^{m, s}(k, u)=\langle k, u, m ; s, \lambda \mid \psi\rangle
$$

or equivalently

$$
|\psi\rangle=\sum_{\lambda} \int \frac{d^{3} k}{2 \omega(\underline{\vec{k}})} \psi_{\lambda}^{m, s}|k, u, m ; s, \lambda\rangle
$$


The scalar product is given by

$$
\langle\varphi \mid \psi\rangle=\sum_{\lambda} \int \frac{d^{3} \underline{\underline{k}}}{2 \omega(\underline{\underline{k}})} \varphi_{\lambda}^{* m, s}(k, u) \psi_{\lambda}^{m, s}(k, u) .
$$

Now we can identify the wave functions related to the localized states (86); namely, we have

$$
\chi_{\lambda}^{m, s}(\vec{\xi}, \tau, k, u ; \sigma ; t)=\frac{1}{(2 \pi)^{3 / 2}} \sqrt{u k} e^{i k_{\mu} \xi^{\mu}} \delta_{\sigma \lambda} .
$$

It follows that in this realisation

$$
\hat{x}^{i}=-i \frac{\partial}{\partial k_{i}}+\frac{1}{2} i\left(\frac{u^{i}}{u k}-\frac{k^{i}}{(u k)^{2}}\right) .
$$

Evidently, for $\xi^{0}=0$ (i.e. for $t=\tau$ ) the functions $\chi$ are eigenvectors of $\hat{x}^{i}$. It can be easily demonstrated that in the preferred frame $(u=(1, \overrightarrow{0}))$ the function (92) reduces to the Newton-Wigner localized state (83); then also the operator (93) coincides with the Newton-Wigner position operator (84) for a spinless particle.

\subsection{Invariant measure}

In the previous sections we used the Lorentz invariant measure (59)

$$
d \mu(k, m)=d^{4} k \theta\left(k^{0}\right) \delta\left(k^{2}-m^{2}\right) .
$$

We point out that this measure is not invariant under the action of the operator $e^{-i q \hat{\vec{x}}(u)}$ (compare eq. (55)). Nevertheless it is possible to find the measure which is both Poincaré invariant and invariant under the action of this operator. One can easily check that such a measure can be written as

$$
d \bar{\mu}(k, m)=u k d \mu(k, m)=u k d^{4} k \delta\left(k^{2}-m^{2}\right) \theta\left(k^{0}\right) .
$$

Our motivation to introduce the measure (94) is that it simplifies some of the formulas discussed herein and resembles the nonrelativistic one. Let us integrate the measure $d \bar{\mu}(k, m)$ with respect to the $k_{0}$. We find

$$
\int d \bar{\mu}(k, m) f(k)=\frac{1}{2 u^{0}} \int d^{3} \underline{\rightarrow} f\left(k_{0}, \underline{\rightarrow}\right),
$$

where $k_{0}$ is given by (53). Now the normalization (64) is not invariant under

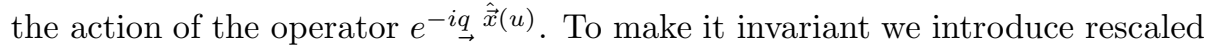
basis vectors

$$
|k, u, m ; s, \lambda\rangle_{i n v}:=\frac{1}{\sqrt{u k}}|k, u, m ; s, \lambda\rangle .
$$


The rescaled vectors are normalized as follows:

$$
{ }_{i n v}\left\langle k, u, m ; s, \lambda \mid k^{\prime}, u, m ; s^{\prime}, \lambda^{\prime}\right\rangle_{i n v}=2 u^{0} \delta^{3}\left(\stackrel{k}{\rightarrow}-\underline{k}^{\prime}\right) \delta_{s s^{\prime}} \delta_{\lambda \lambda^{\prime}} .
$$

This normalization is invariant under the action of the operator $e^{-i q} \stackrel{\vec{x}(u)}{\rightarrow}$ and it is simultaneously Lorentz invariant. Moreover

$$
e^{-i q \underline{\hat{x}}(u)}|k, u, m ; s, \lambda\rangle_{i n v}=e^{i q_{0} t}|k+q, u, m ; s, \lambda\rangle_{i n v},
$$

where $q_{0}=q_{0}(k, q, u)$ is given by (56). The action of the operator $U(\Lambda)$ on the rescaled basis vectors has again the form (60), i.e.

$$
U(\Lambda)|k, u, m ; s, \sigma\rangle_{i n v}=\mathcal{D}_{\sigma \lambda}^{s}{ }^{-1}\left(R_{\Lambda, u}\right)\left|k^{\prime}, u^{\prime}, m ; s, \lambda\right\rangle_{i n v} .
$$

Now let us return to the position operator and localized states. The localized states can be expressed in the new basis as follows

$$
|\vec{\xi}, \tau, u, m ; s, \lambda ; t\rangle=\frac{1}{(2 \pi)^{3 / 2}} \frac{1}{2 u^{0}} \int d^{3} \underline{\rightarrow} e^{i k_{\mu} \xi^{\mu}}|k, u, m ; s, \lambda\rangle_{i n v},
$$

where $\xi^{0}=\tau-t$. The corresponding wave functions localized at the time $t=\tau$ take the form

$$
\tilde{\chi}_{\lambda}^{m, s}(\vec{\xi}, \tau, k, u ; \sigma ; t)=\frac{1}{(2 \pi)^{3 / 2}} e^{i k_{\mu} \xi^{\mu}} \delta_{\sigma \lambda},
$$

and the corresponding position operator takes the extremely simple form

$$
\hat{x}^{i}=-i \frac{\partial}{\partial k_{i}}
$$

like in the nonrelativistic case.

\section{Conclusions}

According to suggestions of some authors (Bell [5], Eberhard [8]), that a consistent formulation of quantum mechanics demands existence of a preferred frame, we constructed here a quantum mechanics which has built-in the preferred frame and which is at the same time Poincaré covariant. We used introduced in [19] a nonstandard realisation of Poincaré group; in this formulation the boost matrix has the lower-triangular form so the time coordinate rescales only under Lorentz transformations. Such a realisation corresponds to a nonstandard synchronization of clocks (CT synchronization) i.e. to a different than standard coordinate time definition. Clasically such a scheme is operationally indistinguishable from the standard one. Our construction shows, that some notions (like causality, localizability) are simultaneously compatible with quantum mechanics and Poincaré covariance only if we resign with democracy between inertial frames, i.e. if a privileged frame is distinguished. In this formulation of 
QM causal problems connected with violation of Bell's inequalities disappear. Quantum theory remains in such a framework non-local but it is causal. In this context we constructed and classified unitary orbits of the Poincare group in the appriopriate bundle of Hilbert spaces. The unitary orbits are classified with help of mass and spin, similary as for the standard unitary representations of the Poincaré group, howeover are induced differently from $S O(3)$. We introduced a Poincaré covariant algebra of momentum and position operators satisfying all fundamental physical requirements. We proved that in our framework the position operator exists for particles with arbitrary spin. It fulfills all the requirements: it is Hermitean and covariant, it has commuting components and moreover its eigenvectors (localised states) are also covariant. We found the explicit functional form of the position operator and demonstrated that in the preferred frame our operator coincides with the Newton-Wigner one. We also defined a covariant spin operators and constructed an invariant spin square operator. Moreover, full algebra of observables consisting of position operators, fourmomentum operators and spin operators is manifestly Poincaré covariant in this framework. We hope that this formulation may be usefull in the construction of the Poincaré covariant version of the de Broglie-Bohm quantum mechanics as well.

\section{Acknowledgements}

This work was supported by the University of Łódź grant no. 621.

\section{References}

[1] A. Aspect, J. Dalibard, G. Roger, Phys. Rev. Lett. 49, 1804, (1982).

[2] A. Aspect, P. Graingier, G. Roger, Phys. Rev. Lett. 47, 460, (1981).

[3] A. Aspect, P. Graingier, G. Roger, Phys. Rev. Lett. 49, 91, (1982).

[4] H. Bacry, "Localizability and Space in Quantum Physics", Springer-Verlag Lecture Notes in Physics Vol. 308, Springer-Verlag, Berlin, Heidelberg 1988.

[5] J. S. Bell, "Quantum mechanics for cosmologists" in "Quantum Gravity", eds. Isham, Penrose and Sciama, Oxford 1981, p. 611.

[6] J. S. Bell, Phys. Rep., 137, 9 (1986).

[7] J. S. Bell, Speakable and Unspeakable in Quantum Mechanics, Cambridge University Press, Cambridge 1987.

[8] P. H. Eberhard, Nuovo Cim., 46B, 392 (1978). 
[9] P. R. Holland, The quantum theory of motion, Cambridge University Press, Cambridge 1993.

[10] R. Hughes, The Structure and Interpretation of Quantum Mechanics, Harward University Press, Harward 1989.

[11] C. J. Isham, Quantum Theory: Mathematical and Structural Foundations, Imperial College Press 1995, Distributed by World Scientific, Singapore.

[12] M. Jammer in Problems in the Foundations of Physics, North-Holland, Bologne, 1979.

[13] R. Mansouri, R. V. Sexl, Gen. Relativ. Gravit. 8, 497 (1977).

[14] T. D. Newton i E. P. Wigner, Rev. Mod. Phys., 21, 400 (1949).

[15] J. Niederle, "Localizability of particles" in Proceedings of the Conference on Hadron Constituents and Symmetry, Smolenice 1976, Physics and Applications Vol. 3, Veda, Bratysława 1978.

[16] A. Peres, Quantum Theory: Concepts and Methods, Kluwer Academic, Boston 1993.

[17] H. Reinchenbach, Axiomatization of the Theory of Relativity University of California Press, Berkeley, CA, 1969.

[18] J. Rembieliński, Phys. Lett. A78, 33 (1980).

[19] J. Rembieliński, Int. J. Mod. Phys. A12, 1677, (1997), hep-th/9607232.

[20] J. Rembieliński i P. Caban, "The Preferred Frame and Poincaré Symmetry" in Physical Applications and Mathematical Aspects of Geometry, Groups and Algebras eds. H. D. Doebner, W. Scherer, P. Nattermann, World Scientific, Singapore 1997, p. 349, hep-th/9612072.

[21] H. P. Stapp, Nuovo Cim., 40B, 191 (1977).

[22] C. M. Will, Phys. Rev. D45, 403 (1992).

[23] C. M. Will, Theory and Experiment in Gravitational Physics, Cambridge University Press, 1993. 